\title{
LOW INTENSITY BEAM DIAGNOSTICS WITH MICROCHANNEL PLATE DETECTORS
}

\author{
G. Cuttone*, C. De Martinis**, P. Finocchiaro*, D. Giove**, G. Raia*, A. Rovelli* \\ *INFN-LNS, Via Santa Sofia, Catania - Italy \\ **INFN-LASA and University of Milan, Via Flli Cervi 201, Segrate(MI)- Italy
}

\begin{abstract}
A non destructive low intensity ion beam profile monitor has been designed and tested. The system is based on a MCP that detects the residual gas ionization produced by the ion beam. The detector is coupled to a phosphorous screen for the readout of the vertical and horizontal density profiles. Experimental results have shown that the system is able to detect beam intensities as low as $10^{8} \mathrm{pps}$ with a local vacuum in the measuring box of the order of $8 \times 10^{-6}$ mbar. In the paper we report the results so far obtained in beam sensitivity and measure precision as a function of the residual pressure and in different regimes of the detector.
\end{abstract}

\section{INTRODUCTION}

The measurement of low intensity beam profiles is of great interest for the design of accelerator based medical and radioactive beam facilities ${ }^{[1]}$. The typical electrical and optical devices used in beam diagnostic can hardly operate below $10^{8} \mathrm{pps}$. At this end a research program is underway at our laboratory for the development of detectors which sensitivity range could span from $10^{10} \mathrm{pps}$ to at least $10^{5} \mathrm{pps}$, and which have no interference with the beam ${ }^{[2]}$. In this paper we present the results so far obtained in the development of detectors based on microchannel plate coupled to a phosphorous screen that gives a one dimensional optical image of the ionization track in the residual gas. The design of a beam imaging device that allows to produce a $2 \mathrm{D}$ transverse beam profile with a higher sensitivity with respect to the residual gas is also presented. It is still based on a MCP coupled with a scintillating screen, but in this case we collect the secondary electrons produced in the interaction of the beam with a thin carbon foil.

\section{EXPERIMENTAL SET-UP}

A non destructive profile monitor has been designed to quantitatively study the beam transversal distributions and dimensions versus the total current. The experimental setup has been improved also including a Faraday cup for the total beam current measurement before the MCP station and a Chromox 6 alumina screen used as a reference device for the transversal beam dimension determination. The measurement point has been fixed along a beam transport line at LNS (Catania) where Tandem and Cyclotron beams are available.

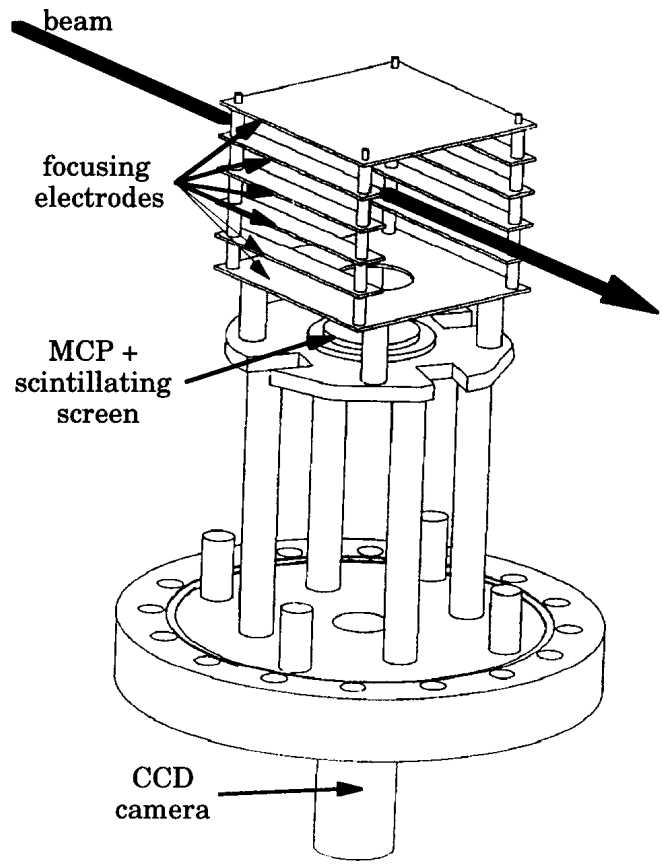

Fig. 1 Sketch of the residual gas beam monitor

A significant limitation of this set-up is represented by the lack of a precise residual pressure control due to the large volumes involved in the detector area. Two different configurations of the beam profile monitor based on microchannel plates coupled to a phosphorous screen have been built. The first one detects the ionization track of the beam on the residual gas. Fig. 1 shows a schematic of this set-up. The electrons or the ions produced on the residual gas are driven to the MCP input by a transverse electric field. The MCP amplified electrons impinging on a phosphorous screen produce an optical image which is strictly correlated with the projection of the beam track on the MCP plane, so providing a one dimension position information. The MCP we used is the F2222-P21 type from Hamamatsu and has an useful dimension of $20 \mathrm{~mm}$ diameter. The readout system is a thin scintillating screen, viewed through a vacuum glass window by a CCD camera. The acquisition of video signals is performed by means of a frame grabber board installed inside a Windows 95 driven PC. The board is a Neotech ISA PC plug-in board with 1 Mbyte of on board memory and the capability to handle timing and trigger information along with video frames. Data acquisition, storage and analysis 
are performed by a LabVIEW based application developed according to the typical requirements for a beam diagnostic tool. The software consists of different modules providing functionality like acquisition and handling of single frames or sequences, image averaging, automatic measurements of beam FWHM. The same analysis software has been used for the management of the images produced by the Chromox 6 screens.

The system previously described has been extensively tested with $\mathrm{C}$ and $\mathrm{Ni}$ beams at $6 \mathrm{MeV} / \mathrm{n}$ and $30 \mathrm{MeV} / \mathrm{n}$ respectively and results are presented in the following.

A second apparatus has been built and it is based on secondary electron emission by thin metallic foils (carbon or aluminium with thickness down to $30 \cdot \mathrm{g} / \mathrm{cm}^{2}$ ). This should enhance by order of magnitude the sensitivity of the system due to the higher yield of electrons produced in comparison with residual gas ionization. Fig. 2 shows a sketch of the system.

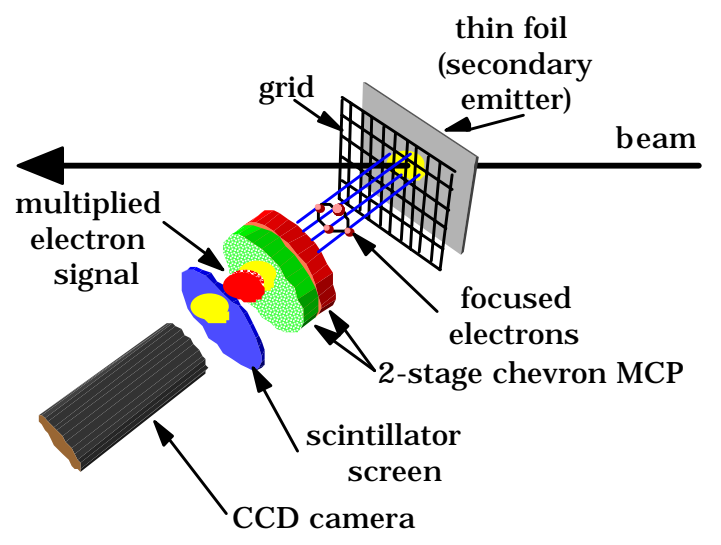

Fig. 2 Secondary electrons emission beam monitor sketch

The secondary electrons produced by the beam interaction are suddenly accelerated to a few $\mathrm{keV}$ of energy by the grid and then driven to the MCP input which is screened from the beam path. In this way the lateral diffusion of the electrons is prevented and in our configuration we calculated that it is less than $0.3 \mathrm{~mm}$. The MCP readout system and image analysis are identical to the one discussed above. We have to point out that this type of detectors can not be considered completely non interfering with respect to the beam because of the stripping action of the foil, unless we do not consider only fully stripped ions.

\section{MEASUREMENTS AND DISCUSSION}

The residual gas beam profile monitor has been extensively tested using a $\mathrm{C}^{5+}$ beam at $6 \mathrm{MeV} / \mathrm{n}$ and a $\mathrm{Ni}^{16+}$ beam at $30 \mathrm{MeV} / \mathrm{n}$. Preliminary measurements have shown that collecting the positive ions at the MCP we have a more defined image in comparison to the electrons collection mode. This behaviour should be due to the lower signal to noise ratio and to a lower lateral diffusion of the ions with respect to the electrons. All the results reported in the following refer to the ion collection mode. Measuring a Carbon beam of $10^{10} \mathrm{pps}$ in a operating vacuum of about $10^{-6} \mathrm{mbar}$ we get the image shown in fig.3.

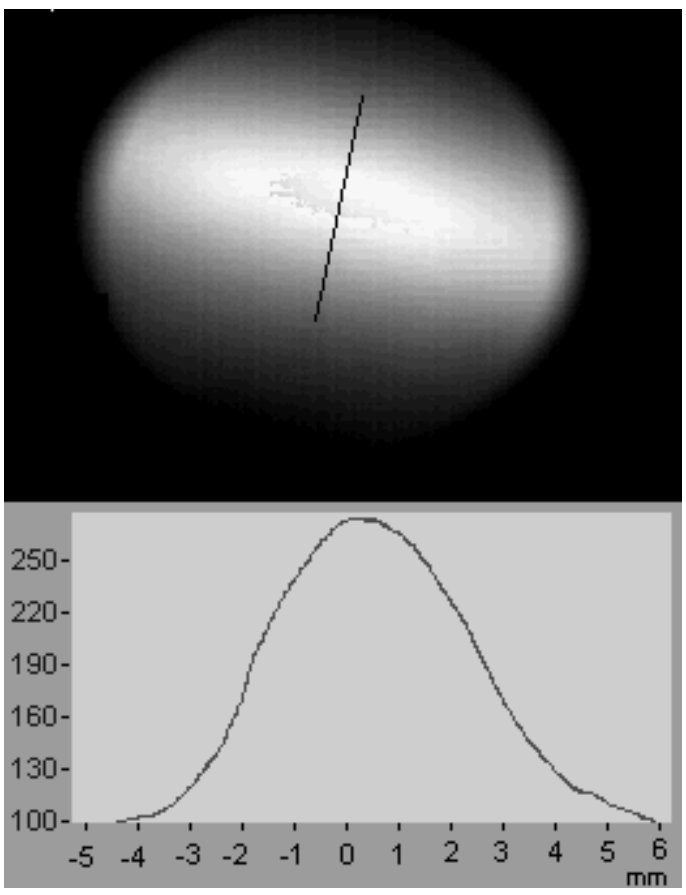

Fig. 3 Trace and horizontal profile with 10 enA of $\mathrm{C}^{+5}$

The measured beam size is about $5 \mathrm{~mm}$ FWHM and the estimated resolution is of $0.3 \mathrm{~mm}$ taking into account the calibration error and the broadening of the ions. The quality of the image is very good and assuming that we have a significant picture if the ratio of the peak value to the background is at least a factor two, we can deduce that the sensitivity of the system is at least of $10^{9} \mathrm{pps}$. This means that if the expected electron-ion production yield ${ }^{[3]}$ is of the order of $2 \times 10^{10}$ pairs $/ \mathrm{mm} / \mathrm{mbar} / \mathrm{pnA}$, neglecting the collection losses we get a production of $3.2 \times 10^{-6}$ pair/particle $/ \mathrm{mm}$. Assuming that the sensitivity of the system is $10^{9} \mathrm{pps}$, we deduce that the limit to the detection of the ionization track is of $3.210^{3} \mathrm{pairs} / \mathrm{mm} / \mathrm{s}$.

More systematic measurements have been done with a $\mathrm{Ni}^{16+}$ beam at $30 \mathrm{MeV} / \mathrm{n}$. In this case the beam was 5 $\mathrm{mm}$ wide (measured also with a Chromox 6 alumina) with intensities of $1 \mathrm{enA}$ and $0.5 \mathrm{enA}$. The nominal pressure in the beam line was $8 \times 10^{-6}$ mbar. As shown in fig. 4 the image is clear and the limit of detection is near to $0.3 \mathrm{enA}$, which corresponds to $10^{8} \mathrm{pps}$. Applying the same considerations done for the carbon beam about the ion-electron pair production, we have, assuming a yield of $9 \times 10^{10}$ pairs $/ \mathrm{mm} / \mathrm{mbar} / \mathrm{pnA}$, that the production rate is $10^{-4} \mathrm{pairs} / \mathrm{mm} /$ particle. In the limit case of $0.3 \mathrm{enA}$, i.e. $10^{8} \mathrm{pps}$, we have a system sensitivity of $10^{4} \mathrm{pairs} / \mathrm{mm} / \mathrm{s}$, a 
value which is very similar to that one obtained for the carbon $\left(3.210^{3} \mathrm{pairs} / \mathrm{mm} / \mathrm{s}\right)$, as we expected.

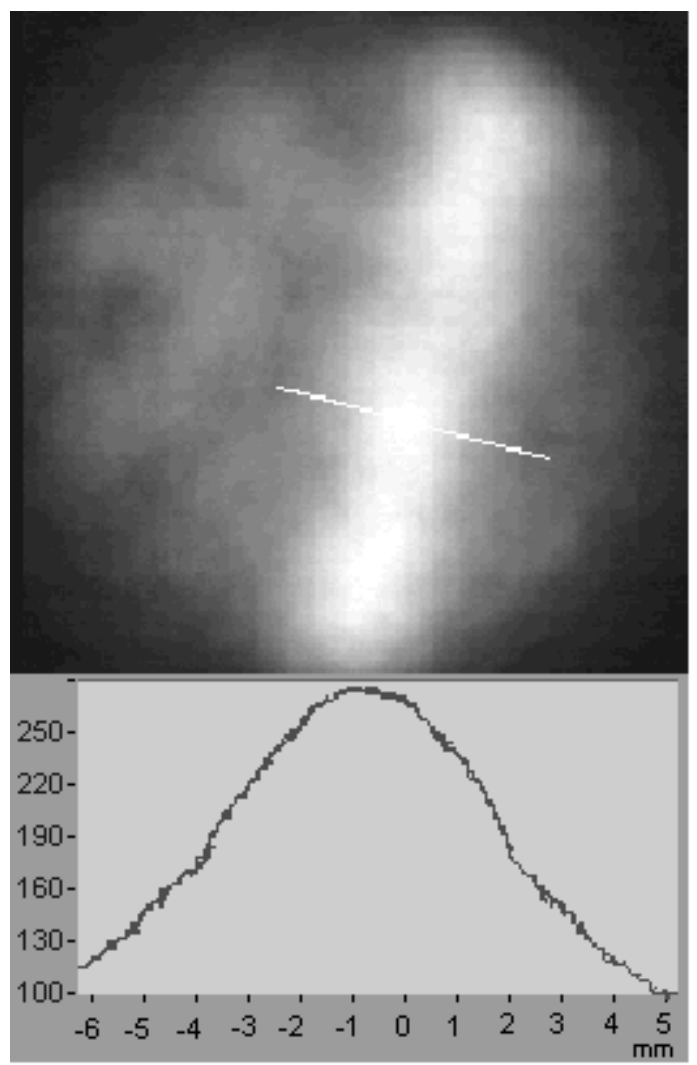

Fig. 4 Trace and horizontal profile with $1 \mathrm{nA}$ of $\mathrm{Ni}^{+16}$

Within a factor three, given by the uncertainties in the knowledge of the true value of the pressure on the MCP region and by the error in the production yield calculation of the electron-ion pairs number in the residual gas, we can assume that the detection limit of our system is given by a production rate of $10^{3} \sim 10^{4} \mathrm{pair} / \mathrm{mm} / \mathrm{s}$ for a beam which is a few millimeters wide.

A preliminary test has also been performed with the secondary electron emission device with a $\mathrm{Ni}$ beam at 30 $\mathrm{MeV} / \mathrm{n}$. A clear beam image was obtained at a very low amplification level of the MCP but, unfortunately, we were not able to continue the measurements for a failure of the MCP.

\section{CONCLUSIONS}

We have constructed two non intercepting beam profile monitors based on the use of MCP. The first one detects the ionization track in the residual gas and has been extensively tested with $\mathrm{C}$ and $\mathrm{Ni}$ beams. The results obtained are interesting and a few comments are in order:

- the technique has proved to be an efficient on line monitor for position, size and beam distribution with beams producing a minimum ionization track in the $10^{3}$ $\sim 10^{4} \mathrm{pairs} / \mathrm{mm} / \mathrm{s}$ range and for a few millimeters diameter spot (with the beams used in our test this means a limit of $10^{9} \mathrm{pps}$ for Carbon and $10^{8} \mathrm{pps}$ for Nickel);

- an increase in the pressure results in a better signal, but the region of $10^{-5} \mathrm{mbar}$ the gain in sensitivity is modest because the noise increases and the MCP is at the limit of its working pressure;

- spatial resolution of $0.3 \mathrm{~mm}$ are obtainable and are well within the requirements for the characterization of the beam.

To increase the detector sensitivity up to $10^{5} \mathrm{pps}$ or less we have realized a second device based on the secondary electron emission by thin metallic foil, but we had no chance to make quantitative measurements with the beam. We plan to do this in the near future and we expect to decrease the lower limit of detection of about two order of magnitude with the same overall performances of the residual gas monitor.

\section{ACKNOWLEDGMENTS}

We should thank Mr. A. Amato for his help in electronics development, Mr. V. Campagna, Mr. C. Marchetta and Mr. S. Salomone for their help in the measurement point assembling.

\section{REFERENCES}

[1] R. Alba et al., Radioactive Ion Beam Facilities at LNS, Proc. RNB III, p. 31, Ed. Frontieres, 1993.

[2] A. Rovelli et al., Low Intensity Beam Diagnostics With Particle Detectors, presented at BIW 96, Argonne.

[3] W. R. Leo, Techniques For Nuclear And Particle Physics Experiments, Springer-Verlag 1987. 